\title{
Regard sur la rencontre animale et pistes méthodologiques
}

\author{
Stéphanie Chanvallon \\ Anthropozoologue, chercheur associé, Laboratoire Violences, Identités, Politiques et Sports (VIPS), Université européenne de \\ Bretagne, 35044 Rennes, France
}

\author{
Mots-clés : \\ relations homme/ \\ animal sauvage ; \\ méthodologie ; \\ anecdote ; ontologie ; \\ éthique
}

\section{Keywords:}

human/wild animal relationships; methodology; ontology; ethics

\begin{abstract}
Résumé - Pour parler de la rencontre d'un homme et d'un animal sauvage, de leurs interactions, il faut surmonter des difficultés d'ordre ontologique, méthodologique et éthique. Des questionnements aux observations sur le terrain, à la compréhension des comportements, entre rationalité et subjectivité, se dessinent des perspectives de recherche au carrefour des sciences sociales et du vivant.
\end{abstract}

\begin{abstract}
A methodology to study encounters with animals. To describe encounters between humans and wild animals and interactions between them, a series of obstacles - ontological, methodological and ethical - needs to be overcome. Out of the questions posed, field observations, and understanding of behaviours, between rationality and subjectivity, opportunities for research may emerge at the crossroads of the social sciences and natural sciences.
\end{abstract}

\section{Qui est celui qui parle de cet autre?}

Dans La dernière harde, Maurice Genevoix écrit la vie d'un cerf nommé le Rouge : « Hier encore, sur l'allée des Mardelles, l'Homme et le chien avaient passé. Ils reviendraient. Le Rouge, maintenant, savait leur opiniâtreté. Leur odeur et leur trace lui devenaient une obsession dont il ne pouvait plus se défaire. Quand ils restaient trop longtemps invisibles, une inquiétude, une angoisse grandissantes, mêlées d'attirance et de crainte, le poussaient à rechercher leurs voies » $(1988$, p. 203). L'intuition de l'auteur donne à voir le monde à travers le regard de l'animal et ferait presque oublier qu'il s'agit là d'une fiction. Mais de quelle réalité parle-t-il ?

Comprendre les interactions entre les mondes animaux - le monde de l'animal sauvage - et humains, « le point de rencontre entre ces deux mondes » (Ellis, 2011, p. 350), les agencements interespèces, est à la fois une quête et une recherche. Quelle méthodologie proposer? Plusieurs questionnements s'imposent en amont : de qui parle-t-on ? Quelle ontologie animale ? D'où parle-t-on, depuis quel socle de perceptions et de connaissances ? À partir du moment où nous nommons, nous mobilisons un ensemble défini de représentations, qu'elles soient scientifiques, sociales, etc., la terminologie permet seulement de dessiner un contour à l'intérieur duquel prennent place des questions de recherche. Toute désignation limite inévitablement le champ d'investigations : pour des scientifiques qui étudient un animal de rente ou de laboratoire, il n'y aura pas de questionnements sur les potentialités animales puisque tel n'est pas leur intérêt, ni d'ailleurs celui des commanditaires. Nous avons donc affaire à des multiplicités de points de vue. Discerner ses propres motivations peut préserver d'une sorte de mauvaise foi, sans oublier qu'au sujet de l'animal et de ses relations avec l'homme, nous ne pouvons faire " comme si » et penser « à la place de ».

La première des questions à poser est presque éthique : « que cherchons-nous vraiment? », soit à quelle question de fond nous voulons répondre. S'agit-il par

Auteur correspondant : stephanie-chanvallon@netcourrier.com

Après un long parcours comme plongeuse professionnelle (biologie marine et réalisation de films documentaires), Stéphanie Chanvallon, anthropologue, s'intéresse aux relations de l'homme avec la nature et tout particulièrement avec les animaux sauvages. Elle mène par ailleurs des recherches en éthologie, s'attachant à la singularité et à l'expérience sensible. 
exemple de débusquer chez l'animal une intériorité proche de la nôtre ? Car nous avons tendance à ne protéger que ce qui nous ressemble ou nous touche au plus près, à l'instar des grands singes. Et lorsque nous trouvons des différences ou des similitudes, qu'en faisonsnous pour les animaux? Lever le voile n'est pas sans conséquences. Il me semble essentiel d'accepter cette introspection d'autant plus pertinente que nous ne sommes pas dans une sociologie ordinaire.

\section{Qu'est-ce que cet « autre » ?}

Il est bien difficile de parler au nom de quelqu'un, alors parler au nom d'un animal est une gageure. Il est courant aujourd'hui de parler des "animaux non humains ", mais dire ceci ne dit pas plus que cela : nous acceptons des parentés. L'intériorité des animaux n'est pas décelable, et ce, d'autant plus que nous cherchons à la démontrer à partir d'outils conceptuels proprement humains rendant l'entreprise quelque peu prétentieuse. «La question concernant le sens donné au comportement des autres n'est pas spécifique aux animaux non humains. L'enquêteur scientifique rencontre le même problème sur la différence quand il a affaire à des enfants, à la folie ${ }^{1}$ » (Morris et al., 2000, p. 152). La difficulté est supplémentaire, les propositions de cette recherche seront, elles, non conventionnelles.

\section{Ontologie, taxonomie... : libérer des vies emprisonnées}

Nous donnons sens au monde à partir du langage mais pas seulement. Nos expériences, nos vécus corporels et sensibles nous permettent d'élargir le champ interprétatif des possibles réalités. À côté de nos représentations du monde animal, la taxonomie, appuyée par l'analyse génétique, classe universellement le vivant. Elle a pour avantage d'en montrer l'extrême diversité, d'accorder précisément les spécialistes sur ce dont ils parlent. Tout ceci selon une échelle croissante de complexité. La comparaison avec le summum de l'évolution sonne parfois comme un couperet entre ce qui a droit ou non à notre faveur ou à notre protection. Aucun être ne peut échapper à cet ordonnancement scientifique, mais tout ne peut y être réduit. La taxonomie a ses limites qui s'ébranlent quand un comportement animal le fait bondir de la case dans laquelle il était installé. D'où l'intérêt et la richesse des recherches non plus in vivo mais in situ, c'est-à-dire sur le terrain de l'animal et non dans l'antre des tables de dissection ou des laboratoires vivants.

\footnotetext{
1 Traduction personnelle : "The question of making sense of the behavior of others is not specific to non-human animals. The scientific investigator encounters the same problem of difference when dealing with children, the insane ».
}

Nous traquons des points de ressemblances, or les animaux ont un comportement qui existe en dehors de nous. Libérer ces vies emprisonnées, c'est, au-delà du sens propre, leur laisser la possibilité d'exprimer autre chose que ce que nous attendons ou supposons pouvoir attendre d'elles. Un animal se définit selon une nomenclature qui permet de le situer dans l'échelle du vivant. Pour exemple, la raie manta, Manta birostris, de l'embranchement des vertébrés, est un poisson cartilagineux de la famille des Mobulidae. Ainsi est reconnu à tel être vivant telle capacité motrice, perceptive, etc. Pourtant notre expérience singulière avec une raie manta, de l'ordre de l'extra-ordinaire, montre qu'il y a là autre chose (Chanvallon, 2013) ${ }^{2}$. Ce qui m'intéresse est le vécu, les comportements des êtres en présence, ce qui s'est passé dans l'instant de la rencontre et qui de toute façon dépasse pour l'animal les considérations liées aux seules sciences naturelles.

«La notion de comportement chère à Merleau-Ponty suffit à rappeler les efforts, aujourd'hui bien connus, de la philosophie contemporaine pour conférer aux autres espèces un statut moral radicalement différent de celui des choses et pour penser les animaux, non pas comme des êtres simplement vivants, mais comme d'autres existences. La vie n'a plus la pauvreté ontologique à laquelle la réduisaient le mécanisme et toutes les philosophies qui concevaient la liberté comme commencement absolu et arrachement à la nature » (Pelluchon, 2015, p. 9).

Avant de mettre en perspective une ontologie animale, il semble opportun de regarder ce que propose aujourd'hui la philosophie comme renouvellement de la pensée, à l'instar de Pelluchon qui, dans sa philosophie $\mathrm{du}$ " vivre de », transforme la perception de l'homme et de son rapport aux autres vivants, en particulier aux animaux (2015).

Comment parler de l'animal ? Ost souligne : « S'arrachant aux déterminations de l'hic et nunc, l'homme est capable de s'universaliser : se mettre à la place de l'autre, de n'importe quel autre» (1995, p. 217). Dans cette ouverture empathique, l'étude de l'animal peut s'envisager à condition qu'elle ne vienne pas servir des intérêts exclusivement humains. Mais un tel désintéressement est-il possible? Si nous devons nous préserver de l'anthropocentrisme, l'anthropomorphisme est depuis longtemps dans la controverse. Gould précise : « Nous ne pouvons nous soustraire au langage et à la connaissance de notre propre expérience affective lorsque nous décrivons une réaction présentant une similitude frappante avec d'autres espèces » (Gould, 2000, cité dans Bekoff, 2009). Pour Bekoff, «L'anthropomorphisme est

\footnotetext{
2 Nous avons nagé avec des dauphins, baleines à bosse..., mais notre rencontre avec cette raie manta a dépassé en intensité et en inattendu, en communion, ce que nous avons pu vivre et partager avec d'autres animaux sauvages.
} 
un phénomène beaucoup plus complexe que nous l'aurions imaginé. Il est fort possible que le besoin humain d'attribuer des émotions aux animaux, loin d'obscurcir leur "véritable nature", puisse être en réalité un outil de connaissance très précis » $(2009$, p. 236). Mais comment reconnaître à l'animal des caractéristiques propres? Peut-être cette évidence est-elle dérangeante car elle nous destitue éthiquement de toute emprise. Faut-il attendre d'une approche anthropomorphique une compréhension sensible? Comment «intuiter» l'animal ? Comment nous perçoit-il, depuis quel monde ? Y a-t-il un décalage entre objectivité et subjectivité, discours et intuition?

\section{Des singularités qui défient le discours scientifique}

La relation homme/animal sauvage est construite sur un attrait commun entre eux. Mais la motivation animale reste insondable, au-delà d'une curiosité évidente. «On se sent face à quelque chose de très grand en termes d'intelligence, de communication. Par le biais de leur attitude, les orques cherchent aussi à communiquer avec nous, ils sont ouverts aux autres espèces, ils ont des interrogations et ils nous interrogent à leur manière ", confie Marc, un enquêté, plongeur apnéiste (Chanvallon, 2013). De plus, il existe toujours des «borderline», des êtres singuliers, atypiques dans leur façon de se comporter. Pour Cardonne, " Quand on part pour observer, il ne faut pas se focaliser sur le sujet choisi, prévu, mais être prêt à voir autre chose... qui souvent se produit » (2004, p. 73). La question de la prophétie autoréalisatrice, pour reprendre la grille de Merton (1968), n'est pas envisagée puisqu'il est alors question d'un événement qui n'est pas prévisible et encore moins connu. Peut-être ne s'agit-il pas de cas isolés mais de ce qui a seulement pu être observé au détour d'une expérience ${ }^{3}$. Si des auteurs ont noté la particularité de certains comportements et en ont souligné le caractère de faible occurrence, ne sont-ils pas significatifs pour autant? L'importance des faits isolés, du côté anecdotique, est preuve suffisante de leur existence. Ces faits sont donc en eux déjà " quelque chose », même si nous ne pouvons à partir de quelques rencontres, à caractère somme toute exceptionnel, avancer une réalité dans le monde animal.

\section{Un animal et un homme dans un agencement}

«[...] il n'y a pas de règne, ni de l'homme ni de la bête, mais seulement des passages, des souverainetés furtives, des occasions, des fuites, des rencontres »

3 Les animaux sauvages se soustraient à nos yeux mais ils ont aussi leur vie sociale, celle dont nous ne sommes que très rarement témoins en milieu naturel - les caméras embarquées sur une fourrure ou dissimulées ici et là dans les herbes et terriers délivrent certes des informations mais elles ne sont qu'une réalité saisie à travers l'œil réducteur de la technologie.
(Bailly, 2007, p. 12). L'animal habite un monde, nous pouvons le partager avec lui. Il est donc essentiel de le considérer dans un agencement. L'agencement naturel est singulier, mouvant, et de fait, la rencontre est unique. Parler d'un animal sauvage sans considérer tout le contexte environnemental, c'est se couper de sources d'informations essentielles à la compréhension de l'événement. Je propose cette approche du «sauvage »: le sauvage n'est pas un espace dénué de présence humaine, il est l'espace des passages du vivant non contraints par les désirs humains ; la fuite est toujours possible. Les animaux captifs sont limités, cloisonnés tant physiquement que psychiquement même si le milieu est organisé pour leur bien-être. Les rencontres et les relations avec l'animal, tout particulièrement lorsqu'il s'agit d'un être sauvage encore peu imprégné par l'homme, s'inscrivent dans un espace aux multiples dimensions et natures. À l'intérieur de l'espace géographique, du jardin à l'océan, se dessine l'espace intime au travers du ressenti, de l'approche, de la distance de fuite jusqu'à la proximité. En effet, l'homme peut découvrir et s'imprégner d'un nouvel espace, le faire sien comme espace identifié de sécurité, le faire évoluer dans la rencontre en fonction de ses appréhensions ou du comportement de l'animal, craintif ou curieux, en attitude de retrait ou de rapprochement. Des congénères à proximité peuvent aussi influer sur le comportement jusqu'à interrompre le lien entre les deux êtres ${ }^{4}$. Évoluer dans un espace de rencontre, c'est créer une relation plus ou moins longue et complexe au sein d'un milieu et de mondes vivants.

Le monde sauvage est un monde de signes, d'émissions de signes qui nous interpellent, nous tiennent en éveil et avec lequel nous pouvons interagir. Quand bien même les prouesses zootechniques accomplissent une réification du vivant annihilant de fait toute possibilité d'expérience de rencontre, quand bien même les zoos mettent en avant une maîtrise du vivant ou une théâtralisation de "nos amies les bêtes", aucun de nos ouvrages et de nos mainmises ne pourra remplacer la spontanéité du vivant. Toutes ces créatures sont dans leurs vies, et ces vies résistent. Ces résistances pourraient déjà éveiller en nous un respect dénué de tout sentimentalisme, un regard originaire. Ainsi, toute classification, toute désignation du vivant, n'en atteindra jamais l'essence et ces possibles expressions. Il y a là quelque chose qui nous échappe, un réseau de vies, de multiplicités et d'agencements auxquels nous appartenons. Nos outils, fondés sur le quantifiable, le disséqué, le penser à la place de, sont une facette, une représentation scientifique, elles valent pour cela, mais seulement pour cela.

\footnotetext{
4 J'ai vécu une telle situation lors d'une rencontre avec une otarie en mer de Cortés. Un mâle est venu la chercher alors que nous étions toutes deux dans un moment de communication par l'imitation mutuelle.
} 


\section{Pistes méthodologiques}

\section{$1^{\mathrm{re}}$ étape : une anthropologie qualitative et réflexive}

Ma méthodologie étant explicitée dans mes précédents travaux (Chanvallon, 2009 ; Chanvallon et Héas, 2011), seules les grandes lignes de la démarche sont présentées ici. Les entretiens au long cours se font auprès d'informateurs privilégiés. Il est question de comprendre qui parle (histoire de vie), ce qui est dit à propos de la relation, de l'animal, depuis quel(s) point(s) de vue. Sont privilégiés dans le discours, la motivation, l'émotionnel, la perception et la sensorialité, l'imaginaire, l'inédit, le réflexif et l'intime, tout ce qui crée du sens au sein et autour des réalités vécues, entre rationalité et interprétation subjective. L'intuition apporte de la flexibilité à la pensée rationnelle. "C'est une logique de l'aléatoire et de l'incertain dont nous avons dès lors besoin pour penser les systèmes ouverts et complexes " (Ost, 1995, p. 246). Il s'agit de saisir un langage apte à formaliser ce qui subjectivement se dit mais qui ne peut toujours objectivement s'expliquer (Alcock, 1981), en sachant qu'au final, «le chercheur développe son discours sur la base de celui des sujets participants et cette rencontre des discours n'est pas sans risque (Caratini, 2004) que l'un ou l'autre vole la parole ou la voit dévoyée. Sachant que le chercheur dispose en général du dernier mot [...], il nous apparaît primordial qu'il réfléchisse à ce pouvoir que le savoir lui confère " (Martineau, 2007, p. 77). À ce travail s'adjoint l'implication du scientifique jusqu'à l'utilisation de ses expériences personnelles comme " matière " pour ses travaux en prenant en considération sa propre trajectoire et tout ce qui pourrait interférer sur les résultats « dans une visée d'élucidation heuristique et d'autonomisation créative de la recherche »(Le Grand, 2006), même s'il est délicat d'espérer une autoanalyse pertinente. Les subjectivités exposées plus loin s'adressent donc à l'enquêté et au chercheur, que celui-ci soit l'enquêteur ou le protagoniste direct dans la rencontre avec l'animal sauvage.

\section{$2^{\mathrm{e}}$ étape : une anthropozoologie des entrecroisements humains et animaux}

\section{Traduire une relation avec un animal sauvage : la solitude du chercheur}

Aller dans une nature non civilisée et rencontrer la vie sauvage, c'est être seul, mais cette solitude n'est qu'une transition entre le monde humain et le monde animal. Interroger la vie sauvage, réfléchir, explorer ce monde, c'est prolonger intellectuellement et psychiquement cette aventure, c'est accepter de marcher sans repères. Cette autre solitude, persistante, dont il faut s'accommoder, vient d'un décalage entre des pensées et des postures scientifiques. Certaines ne s'embarrassent pas de cette problématique de l'animalité ou la récusent parce que l'autre ne dira pas ; fuir le regard est alors une nécessité avant d'être un principe. Traduire une relation avec l'animal implique de percevoir des signes, d'évaluer un ressenti, d'accepter d'avancer sans certitudes, de plonger à l'intérieur de soi avec la résonance du regard animal. Rien ne viendra corroborer des expériences vécues en milieu sauvage parce que nous parlons d'événements, de situations non prévisibles et non reproductibles. Les singularités ne sont pas des exceptions, elles n'apparaissent pas pour disparaître aussitôt. Elles naviguent en périphérie, ont leurs trajectoires, et attendent ou non qu'on leur prête intérêt. Pour les approcher en tant que chercheur, il faut s'engager, résister, accepter les solitudes inhérentes à la position. Étudier une relation atypique entre deux êtres singuliers et leurs comportements, c'est accepter une réflexion tout aussi atypique. Le "work shadowing » ou travail en doublure avec des professionnels (éthologues, vétérinaires, etc.) est possible à condition que la présence sur le terrain d'un tiers ne soit pas un frein à l'immersion du chercheur. Cette situation serait alors à prendre en compte dans les observations réalisées comme interférence possible, tant au niveau humain qu'animal.

\section{Engagement sur le terrain par l'immersion en nature : être aux aguets}

Il est des observations qui ne peuvent être obtenues $q u$ 'aux confins du travail de terrain. Notre éducation et notre culture conditionnent en partie notre sensorialité et notre mode perceptif puis interprétatif (Le Breton, 1990) mais être immergé dans un milieu relativement inconnu, loin de tout repère, instaure une relation nouvelle à l'environnement. Révélateur de l'intime mais aussi de capacités peu explorées dans les modalités de l'ordinaire, être en Nature permet de se dévoiler et d'accéder à des états particuliers où le mental n'est peut-être plus l'unique décideur. L'individu se laisse interpeller par les priorités du moment : vibrer au rythme du milieu naturel, sentir à distance la présence d'un animal, tendre l'oreille, flairer, courber le corps pour se faire discret, suivre des pistes animales, faire silence. Cela redonne de l'importance au corps car son originalité est d'être à la croisée de l'enveloppe individuelle, de l'expérience sociale et du contexte environnemental. Les professionnels qui fréquentent régulièrement un même terrain acquièrent par cette voie une lecture plus fine et immédiate du milieu, ils développent une véritable "stratégie » (Chanvallon et Héas, 2011).

Être aux aguets est aussi une attitude intellectuelle. Nous le sommes dans la traversée des mondes animaux, animés par le désir d'une rencontre, d'un quelque chose qui pourrait nous surprendre. Il y a donc cet « être aux aguets » qui est tension vers, prélude à, pour accueillir 
l'inattendu, l'initiateur d'un autre mouvement de pensée. Ce que nous appelons la folie douce du chercheur caractérise cette attitude qui permet de voir apparaître et peut-être de vivre des expériences singulières, celles qui poussent à l'exploration d'autres territoires, qu'ils soient géographiques ou du psychisme humain. J'acquiesce à ce désir et cette motivation à repousser les limites car « il y a un espoir pour l'humanité » soulignait Gould (1997, p. 278).

\section{Décrire la rencontre}

Pour décrire l'agencement de la rencontre, il faut envisager un nombre conséquent de paramètres. Mais la quantité des questions ne saurait assurer une objectivité. Je peux interroger initialement la nature de la rencontre, est-elle fortuite, désirée, renouvelée ? Dans quelles circonstances se déroule-t-elle? Qui est en présence? Quelles sont les caractéristiques de l'environnement naturel, social, animal ? Quel est le niveau d'interaction en termes d'espace vécu et partagé ? Fuite, curiosité et distance, jeux, contact? Quels signes sont échangés ? Quels sont les modes de communication? Les éléments de la communication non verbale (Hennel-Brzozowska, 2008) sont composés par des signes plus ou moins manifestes : aspect extérieur, comportement spatial - tel que distance interpersonnelle, contact corporel, orientation dans l'espace-, comportement cinétique comme les mouvements du corps, signes vocaux et paraverbaux, silences. Quels sont les modes de relation et d'échanges entre les deux espèces? Sur quoi se fondent-ils, quels sens sont suscités ? Comment sont-ils vécus au niveau de l'affect, par de la peur et un retrait, par une approche et de la curiosité ? Engendrent-ils des interférences sur l'environnement physique ou vivant? Quelles sont les conséquences de ces relations ? Y a-t-il transformation(s) a posteriori, et si oui, de quelle(s) nature(s) sont-elles ? La difficulté réside certes dans la traduction de ce que l'enquêté nous dit de la rencontre mais surtout dans l'interprétation du comportement animal. Un raisonnement anthropocentriste serait insuffisant. Dans ce qui est observable, quelle hypothèse est-il possible de formuler quant au comportement de l'animal ? Témoigne-t-il par exemple d'une curiosité, d'une envie d'entrer en relation, que semble-t-il chercher ? Avec quelle intensité et quelle présence le chercheur va-t-il sentir, observer, vivre par lui-même, traduire la rencontre et la relation à l'animal sauvage?

\section{$\grave{A}$ propos des anecdotes et de l'anthropomorphisme}

Si le travail que nous proposons autour des anecdotes ne peut être comparé avec celui de Renard (2011) en sciences humaines, de toute évidence, nous sommes confrontés à la même défiance en raison de la connotation péjorative qu'elles portent. Cependant, « loin d'être insignifiantes, elles [ces petites histoires] sont au contraire pleines de significations. L'anecdote permet paradoxalement d'aller à l'essentiel » (Renard, 2011, p. 34). Comment l'envisager dans l'étude du comportement animal et de la relation homme/animal sauvage ? "La méthode anecdotique prend de l'ampleur dans l'étude du comportement animal ${ }^{5}$ » (Bates et Byrne, 2007). « [...] dans certains cas, les anecdotes témoignent d'événements significatifs pour lesquels aucune autre méthode ne pourrait se faire l'écho ${ }^{6}$ (Bates et Byrne, 2007). Elles doivent correspondre à « [...] la description originale d'un comportement, à la fois observé par un chercheur ayant une bonne connaissance de l'espèce concernée, et enregistré rapidement dès qu'il est ap$\mathrm{paru}^{7}$ ». Pour qu'elles soient utilisées comme données scientifiques, ils posent trois conditions :

- L'observateur doit être expérimenté, il « sait » de quoi il parle, il connaît l'espèce.

- Pour se préserver de l'anthropomorphisme et de tout autre mésinterprétation, il faut s'en tenir à ce qui est observé.

- Sont utilisées des versions initiales des descriptions (non transformées avec le temps) qui ont elles-mêmes été réalisées juste après l'événement.

De multiples observations indépendantes mais décrivant des phénomènes similaires doivent être recueillies. Les questions concernant l'agencement de l'anecdote, telles que celles posées pour comprendre la situation, me semblent fortement complémentaires. Ainsi, « l'utilisation d'anecdotes nous permet d'appréhender l'étendue des comportements des espèces qui se déroulent en milieu naturel, leurs aptitudes les plus créatives, plutôt que de nous restreindre aux paradigmes déjà répandus sur l'étude des autres espèces animales et des hommes $^{8}$ » (Bates et Byrne, 2007). Il semble judicieux que le chercheur qui relève l'anecdote ait des connaissances sur l'espèce, mais à condition qu'elles ne freinent pas les observations (par exemple, en constituant des attentes de comportements). Comme le souligne Cardonne (2004, p. 109) « c'est le milieu qui détermine son occupant. Car, cela est certain, beaucoup de changements interviendront d'une population à une autre même si la latitude et

\footnotetext{
5 Traduction personnelle: «The anecdotal method is gaining momentum in animal behaviour ».

6 Traduction personnelle : «[...] in other cases, anecdotes reflect low frequency but biologically significant events for which no other method of study is feasible ».

7 Traduction personnelle : « [...] an original description of behaviour, as observed by a researcher well experienced with the species concerned, recorded promptly after occurrence ».

8 Traduction personnelle : « Using anecdotal records allow us to consider the full range of behaviour that occurs naturally in a species, including their most creative aptitudes, rather than restricting the focus to paradigms already familiar from systematic study of other animal species and humans ".
} 
les conditions nous paraissent identiques ». Cela demande donc de la part du chercheur une certaine ouverture et une certaine capacité à accueillir l'anormalité. De plus, la qualité d'une anecdote ne peut être sousestimée sous prétexte qu'elle serait rapportée par un témoin. Tout est question d'attention soutenue, de la qualité de l'immersion dans le milieu de vie de l'animal, au sens propre et figuré ${ }^{9}$.

Si Bates et Byrne veulent se préserver de tout anthropomorphisme, les travaux de Morris et al. (2000, p. 162) apportent des résultats intéressants sur la façon de décrire les comportements des animaux :

« Dans la littérature de la psychologie sociale, la cohérence dans la perception du comportement des autres a été présentée comme une preuve prima facie qu'il doit y avoir quelque chose dans la structure des actions et de la posture humaines spécifique aux différentes intentions et émotions. Sur la base de nos découvertes, nous souhaitons avoir une prétention similaire à propos de la structure du comportement des animaux [...]. Qu'est-ce qui précisément se situe dans la structure des activités humaines et animales et qui permet cette remarquable cohérence dans la description anthropomorphique de ces activités ${ }^{10}$ ".

Pour Bekoff, les résultats de ces recherches signifient : «[...] soit les émotions animales sont plus visibles qu'on ne le croit, soit les humains ont une aptitude naturelle à discerner les émotions des autres espèces » (2009, p. 103). Cependant, dans ces travaux, il s'agit de chiens de compagnie. Est-il possible d'extrapoler à l'observation et à l'interprétation du comportement des animaux sauvages? Dans la même logique, si Morris et al. montrent que la connaissance du chien par les participants n'a pas d'effet significatif sur les résultats, qu'en est-il du savoir de l'observateur sur l'espèce concernée nécessaire dans la méthode de Bates et Byrne? La méthode de la libre détermination du profil mise en œuvre par Wemelsfelder et al. (2000) permet de mettre à jour « des systèmes sémantiques cohérents et signifiants » pour décrire les jugements à l'égard des affects animaux. "La question est de savoir si les gens s'accordent sur les jugements

9 Pour mes recherches sur le comportement exploratoire des orques vis-à-vis des plongeurs en milieu naturel, aller à leur rencontre en apnée était une étape incontournable pour sentir au plus près et accéder à une meilleure appréhension de l'autre.

10 Traduction personnelle: « In the literature on human social psychology, the consistency of the perception of other people's behaviour has been presented as prima facie evidence that there must be something in the structure of human actions and posture specific to different intentions and emotions. On the basis of our findings, we wish to make a similar claim about the structure of animal behaviour [...]. What precisely is it in the structure of human and animal activities that affords such remarkable consistency in the anthropomorphic description of those activities? ». qu'ils portent et s'ils peuvent les employer comme un outil d'évaluation scientifique » (Wemelsfelder et al., 2001, cités dans Bekoff, 2009), qu'il s'agisse, par exemple, de chercheurs expérimentés, d'éleveurs, de vétérinaires, de militants. Dans le cas précis de mon étude sur les interactions orques/plongeurs en milieu naturel, nous pouvons multiplier les regards extérieurs humains venant compléter le vécu du plongeur et les travaux scientifiques. Il pourrait s'agir, par exemple, en suivant la méthode de Wemelsfelder et al., de soumettre des séquences vidéos d'interaction forte avec une orque à différentes personnes ayant ou non une connaissance du milieu marin et de ces animaux et croiser leurs interprétations. Puis de les confronter à des données de recherches. Si nous prenons le cas des émissions de bulles et des secousses de la tête, observées chez les orques captives, sont-elles les signes précurseurs d'une agression chez ces cétacés tels que Turner et Tompkins (1990, cités dans Sarran, 2002) le proposent ? Qu'en est-il en milieu sauvage ? Que disent les plongeurs témoins de ces comportements d'orques observés à quelques mètres devant eux ? À ces pistes méthodologiques, je propose $\mathrm{d}^{\prime}$ adjoindre une bibliographie pluridisciplinaire autour de la pensée écologique en philosophie, de l'interprétation scientifique des systèmes vivants, de la psychologie cognitive, entre autres, pour construire un dialogue entre science et expérience et penser le multiple.

\section{Des subjectivités dans une phénoménologie de la rencontre animale}

Si « [...] un exercice phénoménologique approfondi va révéler avec puissance les limites des interprétations habituelles » (Meyor, 2007, p. 114), alors nous ne pouvons faire l'abstraction d'une phénoménologie de la rencontre animale mais elle est ambitieuse car pouvoir s'approprier cette méthode nécessite, comme toute réflexion construite sur la philosophie, des connaissances particulières et du temps. " Partir du principe que toute conscience est conscience de quelque chose ne fait pas de notre conscience humaine un écran où tout apparaît sur la même fréquence et dans la même tonalité. Trop rares sont donc les analyses qui arrivent à rendre compte de l'expérience dans son caractère vivant, dans son mouvement, dans la tension qu'elle soutient» (Meyor, 2007, p. 115). Mais c'est bien là que se situe le cœur de mon travail : comprendre l'interaction de mondes portés par des animaux d'espèce différente.

Le sujet même de ma recherche ne peut satisfaire à la prétention d'une généralisation des résultats, ni à un groupe, ni à une espèce, ni à une universalité. À partir du moment où j'interroge la relation entre un homme et un animal sauvage dans une situation non prédictible et non reproductible, dans un agencement particulier, les critères de scientificité jusqu'alors donnés ne peuvent 
être satisfaisants. "Ce n'est que dans le cadre d'approches plus périphériques à l'étude du comportement animal que la subjectivité dans son acceptation de création d'un monde de sens propre à l'individu a pu être abordée avec plus de succès » (Calatayud, 2010, p. 328). Les subjectivités avec lesquelles j'ai affaire concernent les êtres en présence et en interaction : l'animal sauvage et l'homme, et le scientifique engagé sur le terrain. Comment aborder la subjectivité ? Peut-on différencier la subjectivité humaine de la subjectivité animale? Doitelle être la pierre angulaire de la réflexion ? Comment penser le comportement anecdotique d'un animal tout en approfondissant la notion de subjectivité comme rapport au monde et donc potentiellement généralisable ? $\mathrm{Si}$ pour Guattari, les processus de subjectivation, « [...] c'est ce qu'il y a de plus riche, de plus hétérogène » (2013, p. 151) et si « la subjectivité a aujourd'hui perdu le goût de la différence, de l'imprévu, de l'événement singulier » (Guattari, 2013, p. 31), alors les subjectivités animales peuvent-elles prétendre à ces ouvertures et répondre à cette singularité ?

Il semble intéressant de mettre en parallèle les travaux d'auteurs cités par Calatayud à propos de l'apparition d'une dimension spatiale et d'un monde de sens et aussi à propos de ce qui lie la perception à l'historique du vécu de l'individu pour donner sens au contexte (André et Baudin, 2002 ; Collot, 2009 ; Varvoglis, 1992). Leurs recherches autour de notions comme les traits de personnalité, la dissonance cognitive, l'état modifié de conscience, la primauté de certaines relations, tout comme l'importance de l'environnement et des espaces vécus (Chanvallon, 2013) apportent des réponses similaires, tout en approchant par une autre dynamique la compréhension des phénomènes. Embrasser ces notions pour comprendre les relations interespèces est un défi pour la recherche par sa prétention à la création et par la prise de risque qui lui est associée. De plus, certains outils permettraient « $\mathrm{d}$ 'illustrer la diversité éventuelle des manières d'être dans une situation donnée [...] Il devient notamment possible de travailler sur un nombre d'individus très restreint sans que cela pose un problème statistique » (Calatayud 2010, p. 336). Dès lors, si «l'éthologie [...] n'est pas l'étude d'un comportement indépendant de l'observateur, mais un discours sur l'être de l'animal au travers de la relation que le chercheur peut avoir avec ce dernier» (Calatayud 2010, p. 335-336), une méthodologie non ordinaire doit être encouragée.

\section{Le multiple et la variation. Le non-prédictible et l'incertain}

Un comportement anecdotique, aussi singulier soitil, est un témoignage privilégié d'un vaste réservoir de possibles. Une seule observation est un résultat en soi, même s'il ne rentre pas dans des bases de données. Nier son existence c'est intellectuellement tricher avec le vivant et se priver de sources d'informations pertinentes. Même si une observation unique ne peut être généralisée, nous ne pouvons en négliger toute la richesse et la laisser de côté ou en suspens. Car " plus un événement est observé et enregistré, plus la possibilité nous est donnée d'en comprendre la fonction et d'en découvrir sa genèse $^{11} »$ (Bates et Byrne, 2007). En milieu sauvage, nous ne pouvons être témoin que d'une infime réalité de ce qui s'y déroule. Or, parmi ces expériences en nature, des situations inhabituelles, atypiques se présentent et viennent semer le doute. Il est nécessaire de leur apporter crédit et de s'y intéresser : la perturbation a en effet quelque chose à nous dire. Au sujet de la diversité en tant que réalité naturelle, Gould souligne : «Nous pensons une tendance comme le mouvement d'une entité dans une certaine direction, alors qu'elle peut être la conséquence secondaire d'un accroissement ou d'une diminution des variations au sein d'un système, de l'ouverture ou de la fermeture de l'éventail des possibles " (1997, p. 49). Il s'agit de considérer les variations du système entier et non de se focaliser systématiquement sur des mesures abstraites que reflète la tendance centrale. De plus, ce qu'il nomme « l'excellence » se développe en de nombreux endroits qu'il est nécessaire d'identifier et de préserver. Tout ce qui donc excelle, sort de l'ordinaire - le fameux contre-exemple - pousse à relativiser et à penser le multiple, l'imprévisible. Dans ces situations singulières, dans ces positionnements en marge, où tout échappe aux prédictions, où tout est rapidement mis entre parenthèses, siège une énergie potentielle de mouvement, de transformation et de devenir.

\section{Pour la créativité. Au croisement des mondes humains et animaux : l'entre-deux animal}

$\mathrm{Au}$ sujet de ces rencontres entre l'être humain et l'animal, Bailly souligne :

« Ce qui est en jeu ici, ce n'est pas l'imitation, par les animaux, de processus mentaux humains, mais c'est une ressemblance étale dont les regards justement sont l'écho un peu comme si en deçà des particularités développées par les espèces et les individus existaient une sorte de nappe phréatique du sensible, une sorte de réserve lointaine et indivise, incertaine, où chacun puiserait mais dont la plupart des hommes ont appris à se couper totalement, si totalement qu'ils n'imaginent même plus qu'elle puisse exister et ne la reconnaissent pas quand pourtant elle leur adresse des signes » (2007, p. 46).

\footnotetext{
11 Traduction personnelle : «the more times an event is observed and recorded, the potential increases for interpreting its function and discovering its ontogeny ».
} 
Dans ces rencontres tellement surprenantes, dans ce surgissement, c'est un autre mode $\mathrm{d}^{\prime}$ " être avec » qui s'impose. Les relations singulières entre un être humain et un animal sauvage poussent les limites de l'altérité, brouillent nos repères et bousculent nos représentations du monde vivant, mais pas seulement. Elles sont aussi vectrices de transformations pour celles et ceux qui les vivent et soulèvent nombre $d^{\prime}$ interrogations quant aux potentialités humaines et animales. Chacun, en fonction de son parcours de vie, de son histoire, de la trame familiale, sociale dans laquelle il s'inscrit et évolue, vit profondément et intimement sa relation à la nature, à l'animal. Nous avons à décrypter et à traduire ce que chacun expérimente, la réalité complexe dans laquelle il s'inscrit et dont il parle. La diversité et la flexibilité ontologique animale associée à l'éventail des sociologies nous invitent à avancer prudemment tout en osant tracer de nouvelles voies. "Quand nous étudions l'intelligence et la créativité des animaux, il est évidemment nécessaire qu'en tant que scientifiques nous soyons créatifs avec nos méthodes ${ }^{12}{ }$ (Bates et Byrne, 2007).

Ce que je nomme «l'entre-deux animal » (Chanvallon, 2014) est l'aboutissement d'une intimité entre deux êtres. Il est la conjugaison d'un agencement et d'un désir partagé, et, de fait, il ne peut se définir qu'en référence à une situation et une seule. Chaque être humain et chaque animal créent ainsi de nouveaux territoires et de nouvelles modalités de relations. Il ne s'agit pas ici d'une expérience conforme à une conception du monde, telles les modalités ontologiques que sont l'animisme ou le naturalisme, mais d'une expérience unique, intime. Cet entre-deux animal, ce mouvement suspendu, est espace de création, presque un art dans l'être lié. Les rencontres des territoires humains et animaux, non seulement géographiques mais psychiques, invitent à l'ouverture de nos frontières. Et si ces territoires se mélangent parfois, il n'y a pas de perte d'identité mais une expérience de l'altérité où percevoir et éprouver produisent du sens par-delà les affects et deviennent une force d'enracinement. Les expériences vécues avec les animaux, nos observations de leurs comportements sont des moments de force, de concentration des formes de vies et d'agencement. Il n'y a pas ici à rechercher une logique mais à accepter des instabilités, des hétérogénéités dont nous sommes spectateurs ou acteurs, et à les faire se rencontrer en maintenant comme question dans ce mouvement de la recherche: qu'est-ce que nous en ferons? "C'est là que l'éthique prend tout son sens : puisque précisément la créativité n'est jamais prise sous des lois, des codes, des règles générales, le processus créatif engage à

\footnotetext{
12 Traduction personnelle: « When studying the intelligence and creativity of animals, it is surely necessary that we as scientists are creative with our methods ".
}

chaque tournant la responsabilité éthique » (Guattari, 2013, p. 552).

\section{$3^{\mathrm{e}}$ étape : Comment penser une éthique efficiente ?}

Pour l'observation et l'étude de la vie sauvage, l'éthique de terrain concerne des règles de bonne conduite à appliquer : ne pas franchir les limites des territoires, ne pas déranger une mère et son petit, ne pas forcer le contact, être à même de percevoir la portée de son intrusion, etc. L'éthique s'attache aussi à une dimension plus vaste. "Le but de l'éthique est que chaque sujet crée chaque jour son propre sens, sa propre façon de devenir plus humain » (Malherbe 2000, cité par Martineau, 2007, p. 71). À propos des recherches qualitatives, Martineau propose cette approche de l'éthique : "L'éthique, en ce début de $21^{\mathrm{e}}$ siècle, repose sur le dialogue entre sujets qui se reconnaissent mutuellement comme sujets [...] le fait de m'adresser à autrui signifie que je nous reconnais une commune appartenance à l'humanité » (2007, p. 72). Comment peut-on prétendre construire une éthique en lien avec l'animal, celle-ci supposant une compréhension initiale, puisque le dialogue avec lui n'est pas possible, puisque toute opinion ne serait que parler à sa place ? La réflexion éthique que je propose interroge non pas un statut moral mais des existences et les relations que ces existences ont entre elles, soit une altérité au sens large indissociable d'une autre réflexion sur la liberté. Elle dépasse les recherches autour de la souffrance des animaux basées sur une hétéroévaluation et qui montrent les difficultés à la reconnaître et à l'apprécier - en sachant qu'une exposition quotidienne à la souffrance peut conduire le chercheur à devenir insensible (Whay et al., 2003, cité dans Le Neindre et al., 2009, p. 154). « [...] nous envisageons de sortir du cadre anthropocentré de l'éthique, en faisant de la protection des espèces qui partagent avec nous l'oikos un devoir " (Pelluchon, 2015, p. 227). Une communauté de liens existe avec les animaux de compagnie et domestiques ${ }^{13}$, mais quid des autres vies ? La réflexion sur l'éthique fait écho aux questions ontologiques et méthodologiques et appelle un engagement réflexif tout en permettant d'identifier ses propres limites. Il n'est pas question de considérer, de respecter ou de protéger un animal en particulier, voire une espèce mais de proposer une ontologie qui pourra avoir une résonance politique. La finalité vise en filigrane la perpétuation et l'épanouissement du vivant sous toutes ses formes au-delà d'une méthodologie de la recherche ayant pour sujet l'animal sauvage. Travailler sur la notion de subjectivité et d'altérité est essentiel.

\footnotetext{
13 Cette communauté de liens est toutefois relative et fonction des usages sociaux. Des animaux appartenant à une même espèce peuvent être traités de façon fort différente selon ces usages : les chats se retrouvent ainsi sur nos genoux ou dans nos assiettes.
} 
Car à la question «qu'est-ce que cet autre? "s'associe «qu'est-ce que être pour cet autre?».

L'éthique est peut-être en lien avec l'esthétisme et le désir parce qu'elle naît non pas de la désignation mais de l'habitation du monde (Chanvallon, 2014). L'émotion esthétique crée une ouverture sur l'extériorité et permet un détachement momentané de soi. "Dans l'art, la contemplation du beau, l'attention aux autres et la communication avec les animaux, nous ne sommes pas coupés de notre intellect, mais nous sommes auprès des choses et du monde" (Pelluchon, 2015, p. 356). Une éthique du monde vivant, une éthique animale, ne pourra être une assertion et de fait répondre d'une obligation morale. Ce qui sera imposé de l'extérieur n'aura pas de prise. Pour faire sens, il faut faire sien, être engagé vers. L'éthique peut être le fruit d'une réflexion et d'une maturation, mais une prise de conscience abrupte peut modifier durablement ou définitivement un rapport au monde vivant, à l'animal. Nous prenons soudainement connaissance de l'existence de l'autre comme altérité et non comme objet ou simple être animé. Une sorte de connexion subtile se réalise. Saisir cette intuition ne se commande pas, ne se veut pas, mais s'impose quand une brèche se fait. Ni statique ni limitée, dans une réflexion critique et un processus réflexif, l'éthique prend sens et s'active par l'accord et la cohérence entre des principes et des actes, le passage illimité de l'un à l'autre. L'éthique $\mathrm{du}$ chercheur appelle efforts, concrétude, autonomie. «Tout discours éthique prétend ainsi être plus qu'un simple discours : il porte en lui la prétention à l'efficience de ce dont il parle » (Bozzi, 2010, p. 15). Ainsi, et toujours selon Bozzi, se pose la question suivante : comment conceptualiser le lien de la pensée éthique à l'acte éthique?

La recherche ne peut être réduite à un pur abstrait trop éloigné du vivant dont elle s'occupe. Pour penser le vivant, une «pensée vivante ${ }^{14}$ » est nécessaire qui fuit les vérités et les représentations courantes, les discours sur les discours, les raccourcis, les évidences, pour laisser vivre ce qui se profile et peut être saisi, pour débusquer de l'incertain ou de l'indécent, pour ne pas dire le vrai mais donner des points de vue, s'offrir aux singularités et questionner encore. Pour Bozzi, « L'éthique organise la fréquentation du monde pour que le désir y coule sans se répandre de façon anarchique [...] c'est la puissance éthique sur l'affect et l'inconscient » (2010, p. 233) un inconscient issu du corps et du social. À la question « qu'est-ce que je cherche? ", la réponse est une curiosité de l'inconnu, une envie d'être saisie par les sens des mondes vivants même si nos sociétés nous ont placés dans des microcosmes dénaturalisés ou presque. Il s'agit

\footnotetext{
14 Voir le texte de Frédéric Bozzi, «Deleuze, une pensée vivante », 2012, disponible sur http:/ / www.fantaisiesdusens. com.
}

d'une curiosité de la première heure, ce qui étymologiquement a soin de, qui est animé par un désir de voir, de comprendre. Habiter le monde, l'altérité, la singularité et le multiple. L'animalité participe de la construction de notre humanité parce qu'elle est différence au sens large et qu'elle nous interroge sur le sens profond de l'altérité comme responsabilité, altération et source de déploiement.

\section{Références}

Alcock J.E., 1981. Parapsychology. Science or magic?, New York, Pergamon Press.

André J., Baudin M. (Eds), 2002. La vie sensorielle. La clinique à l'épreuve des sens, Paris, PUF.

Bailly J.-C., 2007. Le versant animal, Paris, Bayard.

Bates L.A., Byrne R.W., 2007. Creative or created: using anecdotes to investigate animal cognition, Methods, 42, 1, $12-21$.

Bekoff M., 2009. Les émotions des animaux, Paris, Payot et Rivages.

Bozzi F., 2010. Jouer le jeu. Une approche compréhensive de l'efficience éthique, Sarrebruck, Éditions universitaires européennes.

Calatayud F., 2010. Du comportement «fait de nature » au discours de l'éthologiste. Réflexions sur la place de la subjectivité en éthologie, in Burgat F. (Ed.), Penser le comportement animal, Paris, Éditions de la maison des sciences de l'homme/Versailles, Quæ, 323-342.

Caratini S., 2004. Les non-dits de l'anthropologie, Paris, PUF.

Cardonne F., 2004. Approcher l'animal en milieu naturel, Arles, Actes Sud.

Chanvallon S., 2009. Anthropologie des relations de l'Homme à la Nature. La Nature vécue entre peur destructrice et communion intime, Thèse de Doctorat, Université de Rennes 2 HauteBretagne.

Chanvallon S., 2013. Les relations humains/animaux: de l'espace protégé à l'espace partagé, une géographie physique et sensible, Carnets de géographes, 5, janvier, http:/ / www.carnetsdegeographes.org/carnets_recherches/rech 05_01_Chanvallon.php.

Chanvallon S., 2014. Voyage en Nature. Soi, altérité et connivence in Soulancé D., Duhourcau J.-L. (Eds), Quand l'Homme voyage, les passeurs d'empreintes, Paris, L'Harmattan, 183-200.

Chanvallon S., Héas S., 2011. L'Homme et la Nature: enquête/enquête sensible, Natures Sciences Sociétés, 19, 4, 355364.

Collot E., 2009. États de conscience: dissociation simple ou niveaux de réalité ?, in Baud S., Midol N. (Eds), La conscience dans tous ses états. Approches anthropologiques et psychiatriques: cultures et thérapies, Paris, Masson.

Ellis S., 2011. Un homme parmi les loups, Paris, JC Lattès.

Genevoix M., 1988 [1 $1^{\mathrm{re}}$ éd. 1938]. La dernière harde, Paris, Flammarion.

Gould S.J., 1997. L'éventail du vivant. Le mythe du progrès, Paris, Seuil ; traduit de Gould S.J., 1996. Full house. The spread of excellence from Plato to Darwin, New York, Harmony Books. 
Gould S.J., 2000. A lover's quarrel, in Bekoff M. (Ed.), The smile of a dolphin. Remarkable accounts of animal emotions, New York, Discovery Books, 13-17.

Guattari F., 2013. Qu'est-ce que l'écosophie?, Fécamp, Nouvelles Éditions Lignes.

Hennel-Brzozowska A., 2008. La communication non verbale et paraverbale. Perspective d'un psychologue, Synergies Pologne, 5, 21-30.

Le Breton D., 1990. Anthropologie du corps et modernité, Paris, PUF.

Le Grand J.-L., 2006. Implexité : implications et complexité, http:/ / www.barbier-rd.nom.fr/JLLeGrandImplexite.html.

Le Neindre P., Guatteo R., Guémené D., Guichet J.-L., Latouche K., Leterrier C., Levionnois O., Mormède P., Prunier A., Serrie A., Servière J. (Eds), 2009. Douleurs animales. Les identifier, les comprendre, les limiter chez les animaux d'élevage, Rapport d'expertise scientifique collective, Inra.

Martineau S., 2007. L'éthique en recherche qualitative: quelques pistes de réflexion, Recherches qualitatives, horssérie, 5, 70-81.

Merton R.K., 1968. Social theory and social structure, New York, Free Press.

Meyor C., 2007. Le sens et la valeur de l'approche phénoménologique, Recherches qualitatives, hors-série $n^{\circ} 4$, 103-118.
Morris P., Fidler M., Costall A., 2000. Beyond anecdotes: an empirical study of "anthropomorphism", Society $\mathcal{E}$ Animals, 8, 2, 151-165.

Ost F., 1995. La nature hors la loi. L'écologie à l'épreuve du droit, Paris, La Découverte.

Pelluchon C., 2015. Les nourritures. Philosophie du corps politique, Paris, Seuil.

Renard J.-B., 2011. De l'intérêt des anecdotes, Sociétés, 114, 4, 33-40.

Sarran D., 2002. L'apprentissage chez les orques (Orcinus orca) en captivité, Thèse de Doctorat, École nationale vétérinaire de Toulouse.

Turner, T.N., Tompkins, C., 1990. Aggression: exploring the causes and possible reduction techniques, Soundings, 15, 2, 11-15.

Varvoglis M., 1992. La rationalité de l'irrationnel. Une introduction à la parapsychologie scientifique, Paris, InterÉditions.

Wemelsfelder F., Hunter T.E.A., Mendl M.T., Lawrence A.B., 2000. The spontaneous qualitative assessment of behavioural expressions in pigs: first explorations of a novel methodology for integrative animal welfare measurement, Applied Animal Behaviour Science, 67, 3, 193-215.

Wemelsfelder F., Hunter T.E.A., Mendl M.T., Lawrence A.B., 2001. Assessing the "whole animal": a free choice profiling approach, Animal Behaviour, 62, 2, 209-220. 